\title{
ABUNDANCES IN WR NEBULAE
}

\author{
J.M. VILCHEZ and C. ESTEBAN \\ Instituto de Astrofisica de Canarias \\ 38200-La Laguna, Tenerife, Spain
}

\begin{abstract}
Within an ongoing program of long slit spectroscopy of the known WR nebulae, we show here some results for eight objects of the sample selected from the master list of Chu et al. (1983). We present an analysis of their ionisation structure and determine abundances of oxygen, nitrogen and helium in as many positions as possible. The implications of the abundance results for the chemical enrichment of the Interstellar Medium and stellar nucleosynthesis are briefly reviewed.
\end{abstract}

\section{Introduction and Observations}

Of and WR stars are loosing mass at very high rates, exhibiting strong stellar winds which interact with the Interstellar Medium (ISM). According to stellar evolution models, WR stars should be the chemically evolved descendents of massive stars and therefore their stellar winds are expected to be overabundant in helium and heavy elements (e.g. Maeder 1990). Abundances in nebulae sourrounding WR stars can show direct evidence of the chemical processing operating along the evolution of the central star; in particular, WR Ring nebulae offer a unique opportunity to study the chemical and dynamical interaction with the ISM prior to the SN phase.

Most of the abundance studies available for these objects are based on observations of the bright parts of the nebulae or on average spectra without spatial resolution, on the assumption that they are representative of the nebula as a whole (Kwitter 1984; Rosa 1987). However, recent findings suggest that some of these nebulae could be inhomogeneous (Smith et al. 1988; Goudis et al. 1988). Spatially resolved spectroscopy allows sampling zones of the nebulae of differing excitation and (possibly) physical and chemical properties (Rosa and Mathis 1990; Esteban et al. 1990a). Within an ongoing program of long slit spectroscopy of the known WR nebulae, we present here some results for eight objects of the sample selected from the master list of Chu et al. (1983). The observations were intended to fulfil the requirements of wide spectral coverage, spatial resolution and high signal to noise ratios. On the other hand we have also considered necessary for some objects, to combine the preceeding requirements with high spectral resolution in most of the optical range. This method has allowed to isolate different kinematical and/or chemical components, that could be interpreted in terms of central star evolution. 
The observations were performed using the $2.5 \mathrm{~m}$ INT and $4.2 \mathrm{~m}$ WHT at the Observatorio del Roque de los Muchachos (La Palma), and the ESO 3.6m Telescope at La Silla, during different epochs from 1986 to 1990 . Intermediate resolution spectra have typical spectral resolution of $3.7 \AA$ and spatial sampling of 1.5 arcsecs. High resolution observations have typical spectral resolution equivalent to $40 \mathrm{~km} / \mathrm{s}$ FWHM.

\section{Results and Discussion}

\subsection{Ionisation Structure}

The study of the ionisation structure in these objects provides a means of checking the role of photoionisation as the fundamental excitation mechanism, at least for the observed areas within the main body of the nebulae. We claim this test has to be done before any attempt to derive the physical conditions, since the reported evidence of shocked-like characteristics among peripheral zones in some objects (Dufour 1989). In addition, it gives us direct information about chemical peculiarities after comparing with the average normal HII region spectra. For our sample of objects we have used the line ratios diagrams : Log $[\mathrm{NII}] / \mathrm{H} \alpha$ vs. $\log [\mathrm{SII}] / \mathrm{H} \alpha, \log [\mathrm{SII}] \lambda \lambda 6717 / 6731$ vs. $\log \mathrm{H} \alpha /[\mathrm{SII}]$ (Sabbadin et al. 1977) and the series of HII region diagrams from the work of Dopita and Evans (1986). Figure 1 shows two representative diagrams combining [OII],[OIII], and [NII]/H $\alpha$; all the observed points match the locus for normal HII regions in the diagram involving oxygen lines, but this is not the case in the other diagram, where the strength of the observed [NII] lines in some objects deviates clearly from the normal HII region sequence, despite their normal [SII]/H $\alpha$ ratios. This fact is an indication of possible nitrogen overabundances. Overall, the nebulae observed present spectra which are entirely consistent with photoionisation, some of them possibly being nitrogen-rich. Different sets of points located along the sequence in excitation, measured by the $[\mathrm{OII}] /[\mathrm{OIII}]$ and $[\mathrm{OIII}] / \mathrm{H} \beta$ ratios, suggest a range in effective temperatures for the central stars. Effective temperatures for the program nebulae have been ranked according to the diagram in Rosa and Mathis (1990) in which, for some objects in common, we find consistent results.

\subsection{Chemical Abundances}

Ionic and total abundances have been determined for the program nebulae, in as many positions as possible. Direct measurements of the electron temperature have been used in most nebulae though the fainter ones were analysed using the empirical method. Abundances of oxygen, nitrogen and helium mass fraction, Y, are presented in Table 1 for the observed sample and reference objects. For each nebula, spectral type of the central star and estimated galactocentric distance are also quoted. For NGC 2359, S 308, G 2.4+1.4 and NGC 6888 we present average abundances for different zones of the nebulae whose values suggest a non-homogeneous nature. In the case of NGC 6888, the abundances have been taken from the chemo-dynamical study of Esteban and Vilchez (1990), which separates in velocity the spectra of the nebular shell from the ambient component in front of it. Most of the nebulae show $\mathrm{Y}$ and $\mathrm{N} / \mathrm{O}$ values clearly higher than the ones corresponding to their local ISM, being also striking the oxygen-poor nature of a large fraction of them. This result is apparent in Figure 2 where we present $\Delta \log N / O$ vs $\Delta \log O / H$ (defining $\Delta \log =$ $\log _{\text {Nebula }}-\log _{I S M}$, i.e. differential abundance with respect to the ISM according to the galactic abundance gradient), for our sample and for some objects from Rosa and Mathis (1990). In this Figure objects with nearly ISM oxygen abundances $(-0.2 \leq \Delta \log 0 / \mathrm{H} \leq$ 
Figure 1.- $[\mathrm{OIII}] / \mathrm{H} \beta$ vs. [OII]/[OIII] (left) and [OIII]/H $\beta$ vs. [NII]/H $\alpha$ (right) for the WR nebulae sample. Dotted areas correspond to the HII region sequence after Dopita and Evans (1986).
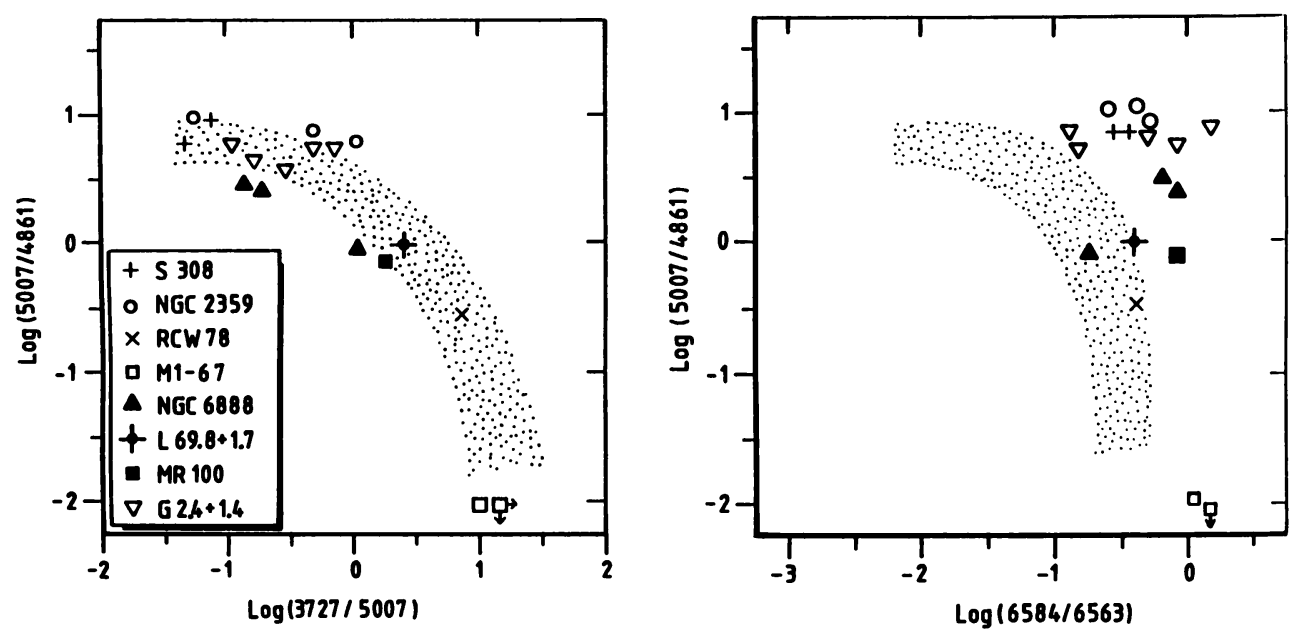

Table 1 .- Average abundances for the observed sample and reference objects

\begin{tabular}{|c|c|c|c|c|c|c|}
\hline Nebula & Sp. Type & $\mathrm{R}_{G}(\mathbf{k p c})^{1}$ & Region & $12+\log O / H$ & $\log N / O$ & $\mathbf{Y}$ \\
\hline \multirow[t]{2}{*}{ NGC 2359} & WN5 & 15 & mean & 8.24 & -0.95 & 0.30 \\
\hline & & & “N streamers" & 8.19 & -0.89 & 0.33 \\
\hline RCW 78 & WN8 & 8.5 & & 8.92 & -0.85 & $\geq 0.31$ \\
\hline$M 1-67$ & WN8 & 9 & & 8.10 & +0.60 & $\approx 0.47$ \\
\hline \multirow[t]{2}{*}{ NGC 6888} & WN6 & 10 & shell & 8.15 & +0.30 & 0.43 \\
\hline & & & ambient & 8.75 & -0.77 & 0.29 \\
\hline \multirow[t]{2}{*}{ S 308} & WN5 & 11 & $\mathbf{A}$ & 8.07 & +0.24 & 0.50 \\
\hline & & & $\mathbf{B}$ & 8.13 & -0.17 & 0.50 \\
\hline MR 100 & WN6 & 10 & & 8.83 & -0.59 & 0.31 \\
\hline L69.8+1.7 & WN8 & 10.5 & & 8.78 & -0.88 & $\geq 0.28$ \\
\hline \multirow[t]{2}{*}{ G2.4+1.4 } & wo & 7 & mean & 8.45 & -0.60 & 0.47 \\
\hline & & & $\mathrm{N}$ filament & 8.46 & -0.25 & - \\
\hline- & & 15 & $<$ HII $>2$ & 8.35 & -1.23 & $0.26^{3}$ \\
\hline- & & 10 & $<$ HII $>^{2}$ & 8.70 & -1.12 & - \\
\hline - & & 8.5 & $<$ HII $>^{2}$ & 8.81 & -1.09 & $0.295^{4}$ \\
\hline - & & 7 & $<$ HII $>2$ & 8.90 & -1.00 & - \\
\hline ORION & & 10 & & $8.60^{5}$ & $-1.00^{5}$ & $0.28^{3}$ \\
\hline LMC & & & & $8.32^{5}$ & $-1.44^{5}$ & $0.25^{3}$ \\
\hline
\end{tabular}

1 Assuming the Sun at $R_{G}=10 \mathrm{kpc}$.

2 Galactic abundance gradient from Shaver et al. (1883).

3 Peimbert (1986).

4 Torres-Peimbert et al. (1989).

5 Rosa and Mathis (1987) and references therein. 
0.2 ) present moderate $\Delta \log \mathrm{N} / \mathrm{O}$ values (within a factor 3 the $\mathrm{N} / \mathrm{O}_{\text {ISM }}$ ) and low $\mathrm{Y}$ fractions $(\mathrm{Y} \leq 0.34$ ), with a single excursion to $\Delta \log \mathrm{N} / \mathrm{O} \approx 0.8 \mathrm{dex}$ for $\mathrm{RCW} 58$ which is a helium rich object (Rosa and Mathis, 1990). On the other hand for oxygen poor objects, the increase in $\mathrm{N} / \mathrm{O}$ correlates well with oxygen defficiency with a maximun around $\Delta \log \mathrm{N} / \mathrm{O}$ $\approx 1.7$ for $\Delta \log \mathrm{O} / \mathrm{H} \approx-0.7$, all these objects having $\mathrm{Y} \geq 0.35$. This result implies an overabundance of $\mathrm{N} / \mathrm{H}$ of at least $0.8 \mathrm{dex}$ for the most oxygen poor nebulae. Within this group are well known nebulae like NGC 6888 and M 1-67; for NGC 6888, shell (S) and ambient (A) points are clearly separated in Figure 2; for M 1-67 the almost pure (80\%) ejecta nature of the nebula seems well established (Esteban et al. , 1990b). The N/H and $\mathrm{O} / \mathrm{H}$ shell abundances for these objects behave so that $\left[X_{O}+X_{N}\right]_{I S M} \approx\left[X_{O}+X_{N}\right]_{\text {Shell }}$ within a factor of two. This behaviour should be investigated for the remaining oxygen poor shells, provided that higher spectral resolution observations are available.

\subsection{Discussion}

The most straightforward result of this work is that processed material, coming from the central star, is certainly present in many nebulae around WR stars. Spectroscopy of WR nebulae in the optical and far-red has been used to derive abundances that, in particular for oxygen, nitrogen and helium, can show variations with respect to the ISM abundance pattern. Evolutionary models for massive stars (Maeder, 1990) predict evolution of surface abundances from the Zero Age Main Sequence to the end of the central carbon-burning phase. Our results qualitatively agree with the overabundances of nitrogen and helium expected from the models, although a more quantitative comparison needs to account for at least the following aspects:

i) Nebular abundances should, in principle, reflect the history of the nucleosynthesis along the evolution of the central star, rather than the predictions of any particular model, therefore only the integrals of models ${ }^{6}$ yields should be compared.

ii) Nebular spectra can be "contaminated" by the ISM; this contamination could act in two ways, a) via real mixing of ejected material and ISM, and/or b) apparent mixture of the nebular spectrum and the spectra of ionised gas in the line of sight (or close) to the nebula, critical for low/intermediate resolution spectroscopy. Both effects, each in a different way, would produce empirical yields which are underestimated.

Bearing in mind these warnings, we have brought together in Figure 3 model predictions and observations, with the aim of just making a crude comparison. In this Figure, the ratio of $\mathrm{N}$ to $\mathrm{O}$ mass fractions, $\mathrm{X}_{N} / \mathrm{X}_{O}$, is represented vs. the helium mass fraction $\mathrm{Y}$. Predictions from Maeder's (1990) models of 15, 25, 40, and $85 \mathrm{M} \odot$ stars of $\mathrm{Z}=\mathrm{Z}_{\odot}$ are shown, up to the WN phase, by line-connected symbols; nebular measurements from our work and that by Rosa and Mathis (1990) are also shown. It is apparent from the Figure that objects with $\mathrm{Y} \leq \mathbf{0 . 3 4}$ converge, like model predictions, to solar abundances with a spread lower than a factor 3 in $\mathrm{X}_{N} / \mathrm{X}_{O}$, and therefore they can be interpreted as almost non-mixed wind blown bubbles or alternatively as extremely diluted ejected material; both interpretations mean in practice that their gas should be undistinguishible from pure wind blown ISM. For helium rich objects two kinds of behaviour are present; on the one hand the nebulae (and $\mathrm{N}$-enhanced zones of nebulae) presenting high $\mathrm{X}_{N} / \mathrm{X}_{O}$ ratios $(\operatorname{say} \geq 1)$ : 
M 1-67, NGC 6888 (Shell) and S 308 A, which can be understood as moderately diluted pre-WN ejected material. On the other hand, those objects showing moderate $\mathrm{X}_{N} / \mathrm{X}_{O}$ values (between 0.2 and 1.) : RCW 104, RCW 58, G2.4+1.4 and S 308 B, which cannot be reproduced by reasonable dilution of ejected material; substantial contamination from ionised ISM in their line of sight could underestimate N/O but this effect can only be operating in crowded environments (perhaps in the direction of G2.4+1.4). For these last objects, a more promising hypothesis can be that of a spatial admixture of N-rich filaments, moving at a different velocity, as has already been suggested for RCW 58 (Smith et al., 1988) and RCW 104 (Goudis et al., 1988).
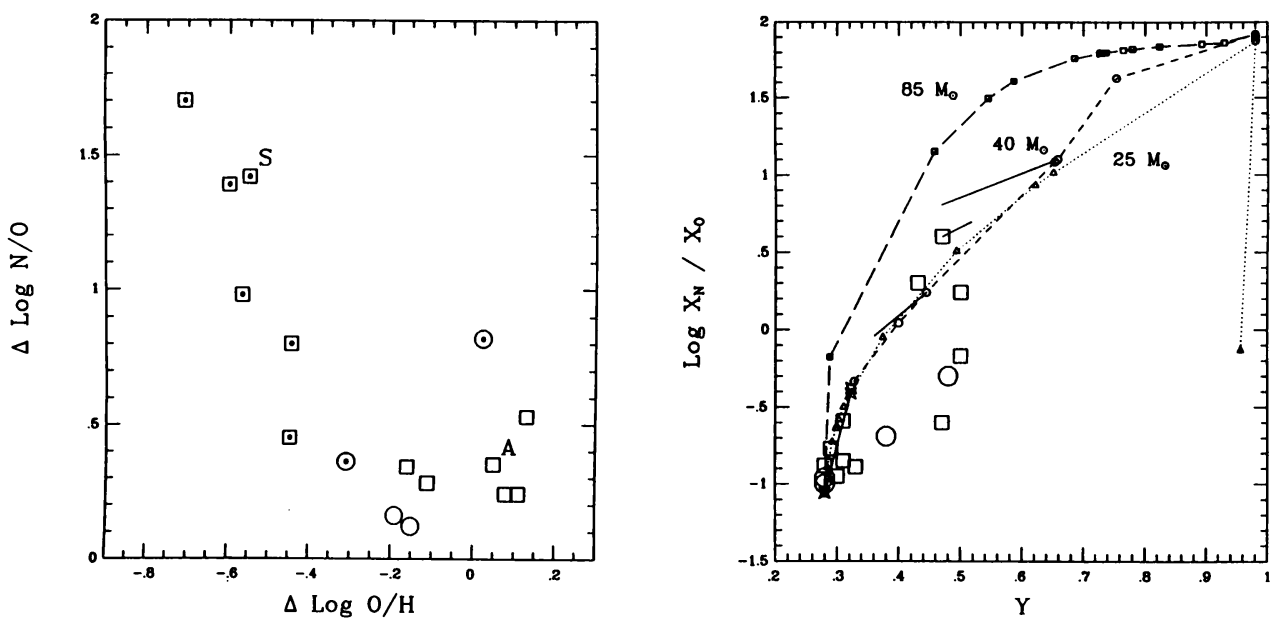

Figure 2 (left).- Differential N/O vs. $\mathrm{O} / \mathrm{H}$ abundances for the sample nebulae; squares: this work, circles: Rosa and Mathis (1990). Symbols of helium rich objects ( $Y \geq 0.34)$ are marked by a black dot.

Figure 3 (right).- The ratio of nitrogen to oxygen mass fractions vs. the helium mass fraction. Lines represent model predictions (Maeder, 1990); symbols as in Figure 2. The effect of a $50 \%$ dilution with the ISM is showm for the $40 \mathrm{M} \odot$ model by a continuous line. 


\section{References}

Chu,Y.-H., Teffers, R.R., Kwitter, K.B. 1983, Ap. J. Suppl., 53, 937.

Dufour, R.J. 1989, Rev. Mexicana Astron. Astrof., 18, 87.

Dopita, M.A., Evans, I.N. 1986, Ap. J., 307, 431.

Esteban, C., Vilchez, J.M. 1990, these proceedings.

Esteban, C., Vílchez, J.M., Manchado, A., Edmunds, M.G. 1990a, Astr. Ap., 227, 515.

Esteban, C., Vilchez, J.M., Smith, L.J., Manchado, A. 1990b, in

preparation.

Goudis, C.D., Meaburn, J., Whitehead, M.J. 1988, Astr. Ap., 191, 341.

Kwitter, K.B. 1984, Ap. J., 287, 840.

Maeder, A. 1990, Astr. Ap. Suppl., 84, 139.

Rosa, M.R. 1987, in Circunstellar Matter, ed. I. Appenzeller and C.

Jordan, Reidel, Dordrecht, p. 457.

Rosa, M.R., Mathis, J.S. 1990, in Properties of Hot Luminous Stars:

Boulder-Münich Workshop, ed. C. D. Garmany, Pub. A. S. P. Conf. Ser.

7, 135.

Sabbadin, F., Minello, S., Bianchini, A. 1977, Astr. Ap., 60, 147.

Smith, L.J., Pettini, M., Dyson, J.E., Hartquist, T.W. 1988, M. N. R. A. S., 234, 625.

\section{DISCUSSION}

Schmutz: (1) What energy distribution did you adopt for the ionizing radiation field? (2) And how sensitive are your abundance determinations to this assumption, especially the helium abundance?

Vilchez: Photoionization model predictions are relatively sensitive to the precise input atmospheres but, more strongly, to the abundances adopted for the gas and to atomic parameters - nominally, in the case of the $S^{+} / S^{++}$ratio, to the ionization cross sections taken by the "model makers"-. Our derived abundances are based on observational electron temperature of the gas, so normally we do not apply model nebulae. I should point out however that, in the case of helium, for nebulae with cool stars the total helium abundance could be underestimated - this is not the case of hot ionizing stars -. This problem is general to OB ionizing stars also! (E.g., Vilchez \& Pagel, 1988; Vilchez, 1989). It is for cool-ionizing star nebulae that models could help best.

van der Hucht: You suggested that the observed abundances of WR ring nebulae could be used as input for WR atmosphere models. But, do you not think that the abundances in WR ring nebulae reflect pre-WR phases (RSG or LBV)?

Vilchez: I suggest that selfconsistent photoionization models for WR nebulae should adopt gas abundances accordingly with the observations. Whenever possible, input star models have to be chosen to correspond to the metallicity of the zone of galaxy where the nebula is located (i.e., $Z_{\odot}, 0.5 Z_{\odot}$, etc.). Of course, I agree that the abundances measured could reflect pre-WR phases, even those often result from a mixture of pollution by the star and ISM. 